\title{
Corynebacterium mucifaciens
}

National Cancer Institute

\section{Source}

National Cancer Institute. Corynebacterium mucifaciens. NCI Thesaurus. Code C122274.

A species of aerobic, non-motile, non-spore forming, non-partially acid-fast, grampositive, coryneform bacteria in the family Corynebacteriaceae. C. mucifaciens is catalase positive, nonfermenting, nitrate reduction negative, and urea and esculin hydrolysis negative; acid was produced from glucose but not from maltose, mannitol, and xylose. The species is nonlipophilic and negative for the CAMP reaction. 